\title{
Predictive significance of the proportion of ER-positive or PgR-positive tumor cells in response to neoadjuvant chemotherapy for operable HER2-negative breast cancer
}

\author{
TOMOFUMI OSAKO ${ }^{1}$, REIKI NISHIMURA ${ }^{1}$, YASUHIRO OKUMURA $^{1}$, YASUO TOYOZUMI $^{2}$ and NOBUYUKI ARIMA ${ }^{2}$ \\ Departments of ${ }^{1}$ Breast and Endocrine Surgery, and ${ }^{2}$ Clinical Pathology, Kumamoto City Hospital, Kumamoto 862-8505, Japan
}

Received June 17, 2011; Accepted August 1, 2011

DOI: $10.3892 /$ etm.2011.359

\begin{abstract}
Estrogen receptor (ER) and progesterone receptor $(\mathrm{PgR})$ status are predictive factors for the clinical and pathological response to neoadjuvant chemotherapy for operable breast cancer. However, it remains unclear as to how the proportion of ER-positive or PgR-positive tumor cells affects the response to neoadjuvant chemotherapy. We examined the correlation of the proportion of ER-positive or PgR-positive tumor cells with the clinical and pathological response to neoadjuvant chemotherapy for operable human epidermal growth factor receptor 2 (HER2)-negative breast cancer. From April 2002 to October 2010, 103 patients received neoadjuvant chemotherapy containing epirubicin and taxane in our clinic. A clinical response was observed in 86 (83\%) patients, and a pathological complete response (pCR) was observed in 16 $(16 \%)$ patients. Fourteen $(30 \%)$ of 46 patients with ER-negative tumors achieved pCR and $15(26 \%)$ of 57 patients with PgR-negative tumors achieved pCR. Patients with more than $30 \%$ ER-positive tumor cells or more than 1\% PgR-positive tumor cells did not achieve pCR. No significant correlation was observed between pCR and the menopausal status, tumor size, grade and Ki-67 expression. In univariate analysis, pCR was associated with the ER status $(p=0.001), \operatorname{PgR}$ status $(p=0.0001)$ and chemotherapy regimens $(p=0.03)$. Multivariate analysis revealed that ER and $\mathrm{PgR}$ status were significant factors for $\mathrm{pCR}$, and patients with ER-negative tumors were 18.6 times more likely to achieve pCR than those with greater than or equal to $30 \%$ ER-positive tumor cells ( $p=0.006 ; 95 \%$ confidence interval 2.3-149.9). We demonstrated a predictive significance of the proportion of ER-positive or PgR-positive tumor cells in the response to neoadjuvant chemotherapy for operable HER2-negative breast cancer. ER-negativity $(<1 \%)$ was a significant predictive factor for achieving $\mathrm{pCR}$ in multi-
\end{abstract}

Correspondence to: Dr Tomofumi Osako, Department of Breast and Endocrine Surgery, Kumamoto City Hospital, 1-1-60 Kotoh, Kumamoto 862-8505, Japan

E-mail: osako.tomofumi@cityhosp-kumamoto.jp; tohsako630108@yahoo.co.jp

Key words: breast cancer, estrogen receptor, progesterone receptor, human epidermal growth factor receptor 2 variate analysis. Conversely, patients with more than $30 \%$ ER-positive tumor cells or more than 1\% PgR-positive tumor cells may not achieve pCR.

\section{Introduction}

Neoadjuvant chemotherapy is often used in the treatment of large, operable, locally advanced breast cancers. This therapy successfully reduces tumor size in most patients and may enable them to consider breast-conserving therapy rather than mastectomy (1-6). In addition, it may permit patients with large inoperable tumors to undergo mastectomy. This approach has not provided a survival advantage as compared to postoperative adjuvant therapy $(1,2,4-7)$. However, patients achieving a pathological complete response (pCR) have a substantially improved disease-free survival (DFS) and overall survival (OS) compared to those with residual disease (8-13).

Estrogen receptor (ER) status is an important predictive factor to achieve pCR in neoadjuvant chemotherapy for operable breast cancer (14-18). Several reports have demonstrated that patients with ER-negative tumors $(<10 \%$ ER-positive tumor cells) are more likely to achieve pCR than those with ER-positive tumors for neoadjuvant chemotherapy for operable breast cancer $(14,15)$. Other studies have reported that patients with ER-absent tumors (0\% ER-positive tumor cells) are more likely to achieve pCR than those with ER-positive tumors (the presence of any detectable positive-staining tumor cells) (16-18).

Recently, the definition of hormone receptor positivity has changed. The cutoff point of $10 \%$ for ER or progesterone receptor $(\mathrm{PgR})$ immunohistochemistry has been the global standard until recently. In the 2009 St. Gallen consensus meeting, the panel recommended the inclusion of adjuvant endocrine therapy for almost all patients whose tumors showed evidence of endocrine responsiveness (presence of any detectable ER). Furthermore, the American Society of Clinical Oncology and the College of American Pathologists (ASCO/CAP) recommended that ER and $\mathrm{PgR}$ assays are considered positive when there are at least $1 \%$ positive tumor nuclei in the sample (19).

How the proportion of ER-positive or PgR-positive tumor cells affects the response to neoadjuvant chemotherapy for operable breast cancer remains unclear. The purpose of this study was to examine the correlation between the proportion 
of ER-positive or PgR-positive-staining cells and the clinicopathological response to neoadjuvant chemotherapy. We retrospectively investigated the clinicopathological factors and responses to neoadjuvant chemotherapy for operable breast cancer at the Kumamoto City Hospital, Japan. We eliminated human epidermal growth factor receptor 2 (HER2)-positive breast cancer from our data as the administration of trastuzumab affects the outcome of neoadjuvant therapy (20).

\section{Patients and methods}

Patients and treatments. From April 2002 to October 2010, 103 patients were enrolled in this study. The clinical and pathological characteristics of all patients were obtained from our institutional medical records. All patients were pathologically diagnosed with invasive breast cancer by core needle biopsy. Moreover, they had positive axillary nodes or tumors of sizes $\geq 3 \mathrm{~cm}$ measured objectively by breast ultrasonography. Prior to starting chemotherapy, patients had a good performance status and no metastatic lesions, which was confirmed by chest radiography, bone scanning, abdominal ultrasonography and whole body computed tomography. Tumor size was measured and followed up by breast ultrasonography. Patients undergoing breast-conserving surgery received radiation therapy for the preserved breast. Patients with receptor-positive tumors underwent standard hormonal therapy. Patient characteristics, such as the clinical stage, menopausal status, histological grade, hormone receptor status, chemotherapy regimen, and clinical and pathological responses in the breast, were recorded.

Chemotherapy regimens. From April 2002 to October 2005, 28 patients received 4 cycles of tri-weekly epirubicin $\left(60 \mathrm{mg} / \mathrm{m}^{2}\right)$ and docetaxel $\left(60 \mathrm{mg} / \mathrm{m}^{2}\right)$ concurrently. From April 2002 to October 2010, 75 patients received 4 cycles of tri-weekly 5 -fluorouracil $\left(500 \mathrm{mg} / \mathrm{m}^{2}\right)$, epirubicin $(75$ or $\left.100 \mathrm{mg} / \mathrm{m}^{2}\right)$ and cyclophosphamides $\left(500 \mathrm{mg} / \mathrm{m}^{2}\right)$, followed by 4 cycles of tri-weekly docetaxel $\left(75 \mathrm{mg} / \mathrm{m}^{2}\right)$ or 12 cycles of weekly paclitaxel $\left(80 \mathrm{mg} / \mathrm{m}^{2}\right)$. The patients underwent surgery for the following conditions: after completion of neoadjuvant chemotherapy; when the tumors continued to be progressive under concurrent therapy of epirubicin and docetaxel; when the tumors continued progression after taxane was administered, in cases where therapy with 5-fluorouracil, epirubicin and cyclophosphamide was ineffective.

Evaluation of the response to chemotherapy. We evaluated the clinical response of primary breast cancer and axillary lymph nodes using ultrasonography, according to the Response Evaluation Criteria in Solid Tumors (21): complete response (CR), disappearance of all target lesions; partial response ( $P R$ ), a decrease of $\geq 30 \%$ in the diameter of the target lesion; progressive disease (PD), an increase of $\geq 20 \%$ in the diameter of the target lesion; and stable disease (SD), neither sufficient shrinkage to qualify for PR nor sufficient increase to qualify for PD.

Pathological response was evaluated according to the following definition: pCR was defined as the complete disappearance of cancer cells from the breast stroma. A nonpathological complete response (non-pCR) was defined as the presence of pathological residues of cancer cells in the breast stroma. However, the outcomes of the axillary lymph nodes were not taken into account.

Pathological examination and immunohistochemistry. Pathological evaluation was performed on patients at the Department of Clinical Pathology, Kumamoto City Hospital. Our pathologists analyzed samples obtained by core needle biopsy prior to starting chemotherapy, as well as those obtained from surgical resection. Formalin-fixed, paraffin-embedded tissue blocks were prepared and stained immunohistochemically for the expression of ER and PgR, the HER2 receptor, p53 and Ki-67 (22). The slides were incubated with a diluted anti-ER primary antibody (1:75; Dako, Glostrup, Denmark), a diluted anti-PgR primary antibody (1:700; Dako), a diluted anti-p53 primary antibody (1:50; Japan Tanner, Osaka, Japan) and a HER2/neu oncoprotein antibody (Herceptest; Dako). The Dako EnVision system (Dako EnVision labeled polymer, peroxidase) or the Benchmark XT system (Ventana Medical System, AZ, USA) were used as the detection systems for ER, PgR and HER2.

Investigated parameters included tumor size, lymph node status, histological grade, ER and PgR status, proliferation index (Ki-67), as well as expression of HER2 and p53. The proportion of ER-positive and PgR-positive tumor cells was expressed as a percentage. The positivities of the ER and $\mathrm{PgR}$ were defined as $\geq 1 \%$ according to ASCO/CAP (19). After patient distribution according to the proportion of receptor-positive tumor cells was examined and compared to the distribution of patients achieving pCR, the patients were classified into groups at appropriate cutoff points. $\mathrm{Ki}-67$ values were expressed as the percentage of positive staining cells in each case and were classified into two groups based on the percentage of positive nuclei: $>20$ and $\leq 20 \%$. p53 expression was categorized into three groups: negative (absent or focal staining with $<5 \%$ tumor cells), $1+$ (heterogeneous or focal staining with $>5 \%$ tumor cells) and $2+$ (homogeneous and diffuse staining). HER 2 overexpression was defined as the strong and diffuse membranous staining of tumor cells. HER2-2+ staining was tested by fluorescence in situ hybridization, with a threshold for positive HER2/CEP17 ratio of $>2.0$.

Statistical analyses. The influence of tumoral pre-operative baseline characteristics on the likelihood of achieving pCR was tested using the Chi-square or Fisher's exact test. Independent significance of variables was analyzed using a multivariate logistic regression model with a step-up procedure. Odds ratios, 95\% confidence intervals (CIs) and p-values were estimated from the final model. The Kaplan-Meier method was used for the assessment of DFS and OS. The log-rank test was used to examine the statistical significance of the differences between groups. Statistical analyses were performed using the Statistical Package for the Social Sciences (SPSS) version 18.0 for Windows (SPSS Japan Inc./IBM Company, Tokyo, Japan).

\section{Results}

Patient distribution according to the proportion of ER-positive or PgR-positive tumor cells. From April 2002 to October 2010, 103 patients underwent surgery following neoadjuvant chemo- 


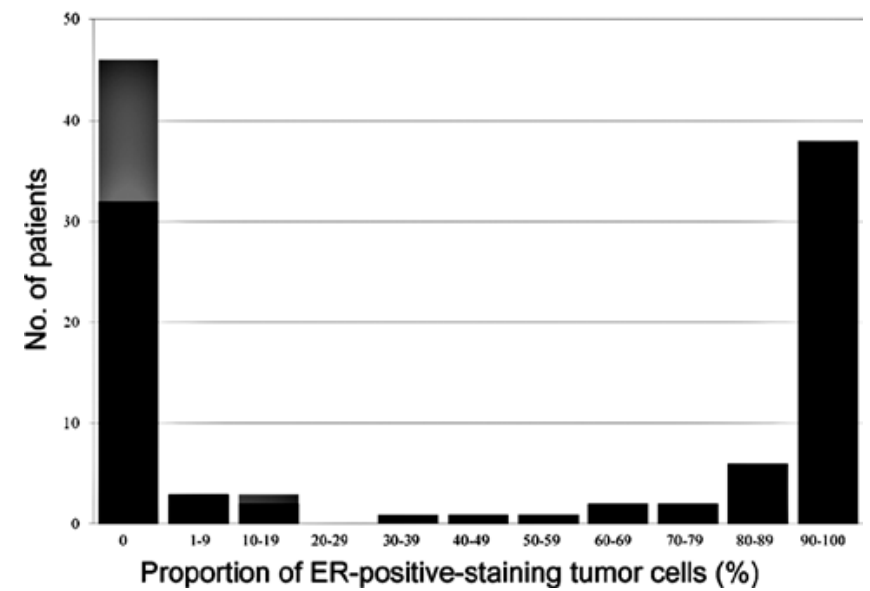

Figure 1. Frequency distribution according to the proportion of ER-positivestaining tumor cells among 103 primary breast cancers. The bar graph shows the bimodal distribution; most of the ER-negative tumors were ER-absent and most of the ER-positive tumors were $>90 \%$ ER-positive. The gray bars indicate the number of patients who achieved pCR. Most of the patients who achieved pCR had ER-absent tumors, and patients whose tumor had $>30 \%$ ER-positive cells did not achieve pCR.

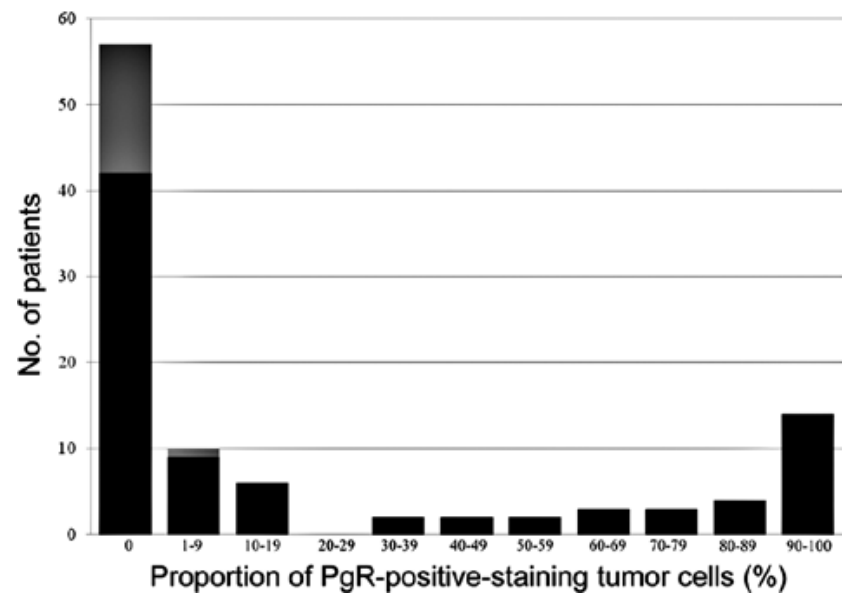

Figure 2. Frequency distribution according to the proportion of PgR-positivestaining tumor cells among 103 primary breast cancers. The gray bars indicate the number of patients who achieved pCR. Most of the PgR-negative tumors were PgR-absent. Most of the patients who achieved pCR had PgRabsent tumors, and patients whose tumor had $>1 \%$ PgR-positive cells did not achieve pCR.

therapy for primary operable breast cancer. Pathological specimens were available in all cases. Of the 103 patients, $46(45 \%)$ were ER-negative and $57(55 \%)$ were ER-positive (Fig. 1). Of the 57 patients with ER-positive tumors, 38 (67\%) had tumors with $\geq 90 \%$ ER-positive cells (Fig. 1). Of the 103 patients, 57 (55\%) were PgR-negative and 46 (45\%) were PgR-positive (Fig. 2).

Response to neoadjuvant chemotherapy. Pathologically, 16 (16\%) patients had no residual disease after chemotherapy. Of 16 patients with pCR in the breast, 3 had positive lymph nodes. Fourteen (30\%) of the 46 patients with ER-negative tumors achieved pCR and 15 (26\%) of the 57 patients with PgR-negative tumors achieved pCR following neoadjuvant chemotherapy (Table I). Although one patient with $30 \%$ ER-positive cells achieved pCR, none of the patients with
Table I. Univariate analysis of factors predicting a pCR according to baseline factors.

\begin{tabular}{|c|c|c|c|}
\hline Baseline factor & pCR, no. (\%) & Total & p-value \\
\hline Total & $16(16)$ & 103 & \\
\hline \multicolumn{4}{|l|}{ ER } \\
\hline Negative & $14(30)$ & 46 & \multirow[t]{3}{*}{0.001} \\
\hline $1-29 \%$ & $1(17)$ & 6 & \\
\hline$\geq 30 \%$ & $1 \quad(2)$ & 51 & \\
\hline \multicolumn{4}{|l|}{ PgR } \\
\hline Negative & $15(26)$ & 57 & \multirow[t]{2}{*}{0.0001} \\
\hline Positive & 1 (2) & 46 & \\
\hline \multicolumn{4}{|l|}{ Clinical tumor size } \\
\hline $\mathrm{T} 1$ & $1(14)$ & 7 & \multirow[t]{4}{*}{0.73} \\
\hline $\mathrm{T} 2$ & $10(16)$ & 63 & \\
\hline T3 & $5(19)$ & 27 & \\
\hline $\mathrm{T} 4$ & $0 \quad(0)$ & 6 & \\
\hline \multicolumn{4}{|l|}{ Nodal Status } \\
\hline Negative & $6(19)$ & 32 & \multirow[t]{2}{*}{0.37} \\
\hline Positive & $10(14)$ & 71 & \\
\hline \multicolumn{4}{|l|}{ Histological grade } \\
\hline Grade 1 & $2(8)$ & 26 & \multirow[t]{3}{*}{0.09} \\
\hline Grade 2 & $6(13)$ & 48 & \\
\hline Grade 3 & $8(28)$ & 29 & \\
\hline \multicolumn{4}{|l|}{ Ki-67 } \\
\hline$<20 \%$ & $0 \quad(0)$ & 11 & \multirow[t]{2}{*}{0.14} \\
\hline$\geq 20 \%$ & $16(17)$ & 92 & \\
\hline \multicolumn{4}{|l|}{ p53 } \\
\hline 0 & $2(15)$ & 13 & \multirow[t]{3}{*}{0.14} \\
\hline 1 & $3(8)$ & 40 & \\
\hline 2 & $11(23)$ & 48 & \\
\hline \multicolumn{4}{|l|}{ Menopausal status } \\
\hline Pre-menopause & $7(12)$ & 60 & \multirow[t]{2}{*}{0.16} \\
\hline Postmenopause & $9(21)$ & 43 & \\
\hline \multicolumn{4}{|l|}{ Regimen } \\
\hline ET & 1 (4) & 28 & \multirow[t]{2}{*}{0.03} \\
\hline FEC-T & $15(20)$ & 75 & \\
\hline
\end{tabular}

ET, concurrent epirubicin and docetaxel; FEC-T, 5-fluorouracil, epirubicin and cyclophosphamides followed by taxane.

$>30 \%$ ER-positive cells or $>1 \%$ PgR-positive cells achieved pCR (Figs. 1 and 2).

All groups demonstrated a good clinical response to neoadjuvant chemotherapy regardless of their ER status (Table II). Out of the 46 patients with ER-negative tumors following neoadjuvant chemotherapy, $16(35 \%)$ achieved CR and 22 (48\%) achieved PR. Out of the 57 patients with ER-positive tumors following neoadjuvant chemotherapy, 8 (14\%) achieved CR and 40 (70\%) achieved PR; a significant difference was observed between them.

Predictive factors for $p C R$. In univariate analysis, $\mathrm{pCR}$ was associated with ER status ( $\mathrm{p}=0.001), \operatorname{PgR}$ status $(\mathrm{p}=0.0001)$ and chemotherapy regimens $(\mathrm{p}=0.03)$ (Table I). No significant 
Table II. Clinical response for neoadjuvant chemotherapy in accordance with ER status.

\begin{tabular}{|c|c|c|c|c|c|c|}
\hline & CR, no. (\%) & PR, no. (\%) & NC, no. $(\%)$ & PD, no. (\%) & Total & p-value \\
\hline ER-negative & $16(35)$ & $22(48)$ & $7(15)$ & $1(2)$ & 46 & 0.045 \\
\hline ER-positive & $8(14)$ & $40(70)$ & $9(16)$ & $0(0)$ & 57 & \\
\hline
\end{tabular}

$\mathrm{CR}$, complete response; $\mathrm{PR}$, partial response; $\mathrm{NC}$, no change; $\mathrm{PD}$, progressive disease.

Table III. Multvariate analysis to identify the baseline factors predicting a pCR.

\begin{tabular}{lccc}
\hline & Odds ratio & $95 \%$ CI & p-value \\
\hline ER status & & & \\
$\quad$ Negative $/ \geq 30 \%$ & 18.6 & $2.3-149.9$ & 0.006 \\
$\quad \begin{array}{l}1-29 \% / \geq 30 \% \\
\text { PgR status }\end{array}$ & 12.8 & $0.6-256.9$ & 0.100 \\
$\quad$ Negative/positive & 14.6 & $1.8-116.4$ & 0.020 \\
$\quad$ Regimen & & & \\
$\quad$ FEC-D/ET & 4.8 & $0.6-41.70$ & 0.160 \\
\hline
\end{tabular}

FEC-D, 5-fluorouracil, epirubicin and cyclophosphamides followed by docetaxel; ET, concurrent epirubicin and docetaxel.

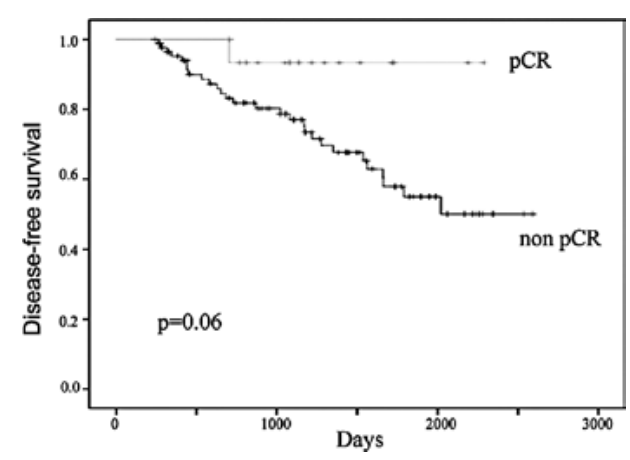

Figure 3. Disease-free survival according to $\mathrm{pCR}$ in all patients.

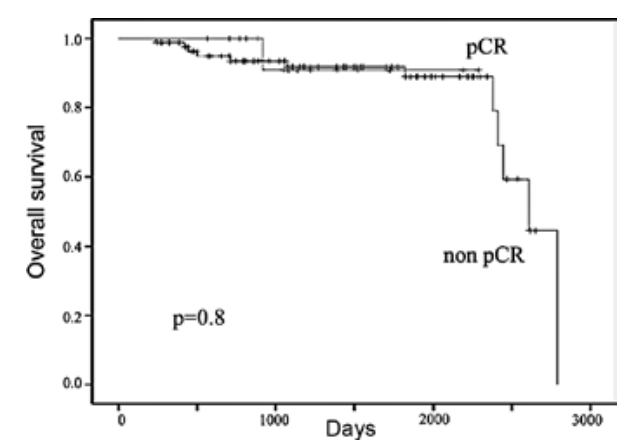

Figure 4. Overall survival according to pCR in all patients.

difference in the pCR rate was observed according to menopausal status, clinical tumor size, nodal status, histological grade and $\mathrm{Ki}-67$ or p53 expression (Table I).

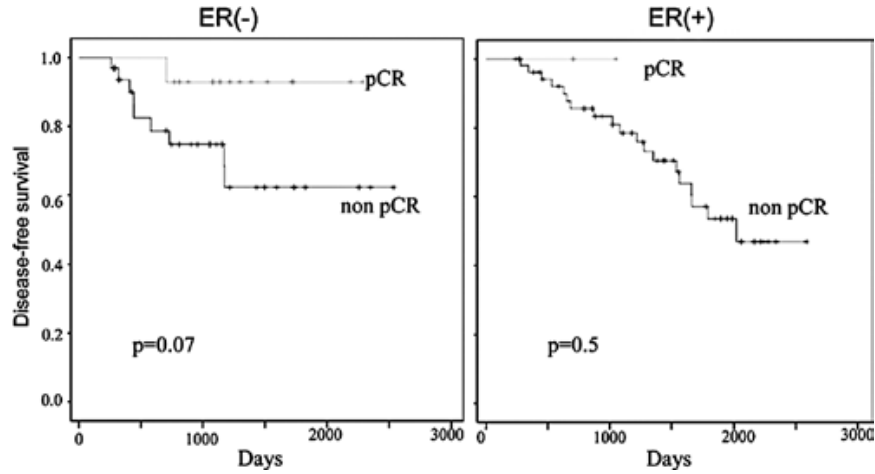

Figure 5. Disease-free survival according to $\mathrm{pCR}$ in patients with ER-negative and ER-positive tumors.
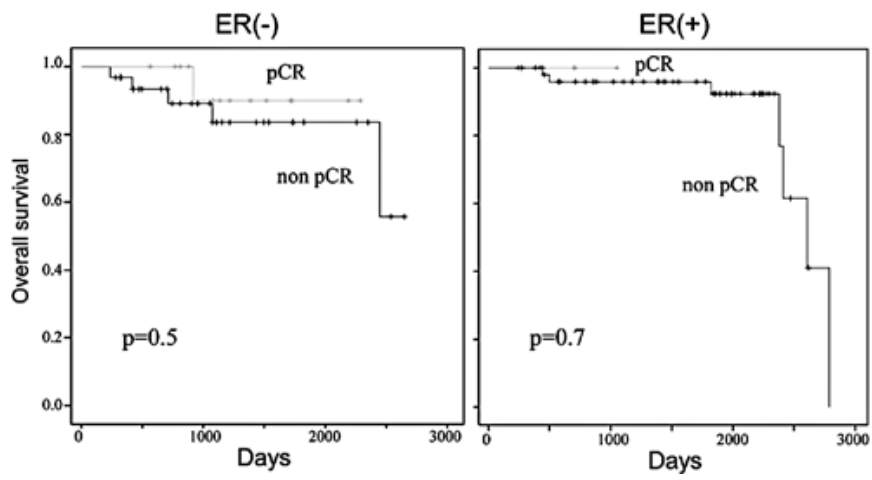

Figure 6. Overall survival according to $\mathrm{pCR}$ in patients with ER-negative and ER-positive tumors.

A multivariate analysis was performed using the ER status, PgR status and chemotherapy regimens. ER and $\mathrm{PgR}$ status correlated with pCR following neoadjuvant chemotherapy using the step-up procedure. Patients with ER-negative tumors were 18.6 times more likely to achieve $\mathrm{pCR}$ than those with $\geq 30 \%$ ER-positive tumor cells ( $\mathrm{p}=0.006$; 95\% CI 2.3-149.9) (Table III).

Survival. At a median follow-up of 40.5 months, the 5-year DFS in all patients was $60 \%$ and the 5 -year OS was $89 \%$. One patient who achieved pCR following neoadjuvant chemotherapy relapsed and died during the follow-up period. The 5-year DFS in patients who achieved and in those who did not achieve pCR following neoadjuvant chemotherapy was 55 and $91 \%$, respectively (Fig. 3). The 5-year OS in patients who achieved and in those who did not achieve pCR following neoadjuvant chemotherapy was 89 and 93\%, respectively (Fig. 4). There were trends in improvement in the DFS of patients who achieved 
pCR, however, they did not reach statistically significant values ( $\mathrm{p}=0.06$; Fig. 3 ). When patients with ER-negative and ER-positive tumors were examined separately, the patients who achieved pCR showed an improved DFS and OS compared to those with residual diseases, but no significant differences were observed between them (Figs. 5 and 6).

\section{Discussion}

We retrospectively investigated several pathological factors to examine the correlation between the proportion of ER-positive or PgR-positive tumor cells and the clinicopathological response to neoadjuvant chemotherapy for HER2-negative operable breast cancer. In multivariate analysis, ER- or PgR-negativity was a significant predictive factor to achieve pCR. Patients with more than $30 \%$ ER-positive tumor cells or more than $1 \% \mathrm{PgR}$-positive tumor cells did not achieve pCR. Most patients achieved a favorable clinical response to neoadjuvant chemotherapy for operable breast cancer regardless of the proportion of ER-positive or PgR-positive tumor cells.

Our results are in accordance with the findings of Colleoni et al that ER-absent tumors were more predictive than ER-low tumors (1-9\%) in achieving pCR following neoadjuvant chemotherapy for breast cancer. They demonstrated that patients with ER-absent and PgR-absent tumors were 12 times more likely to achieve pCR than those with ER-positive and PgR-positive tumors or ER-low tumors (16-18). In addition, they described that approximately two-thirds of ER-negative patients belonged to the ER-absent group, and one-third to the ER-low group. Moreover, the pCR rate of ER-absent patients was $19.4 \%$, but the pCR rate of ER-low patients was $2.8 \%$. On the other hand, other studies related to the predictive factors of neoadjuvant chemotherapy demonstrated that patients with ER-negative tumors were more likely to achieve pCR than those with ER-positive tumors. However, these studies did not distinguish ER-absent patients from ER-low patients among the ER-negative breast cancer patients $(5,9,14,15)$.

In addition, our results suggest that the proportion of ER-positive or PgR-positive tumor cells is a predictive factor for non-pCR in neoadjuvant chemotherapy. Patients with more than $30 \%$ of ER-positive tumor cells or more than $1 \%$ of PgR-positive tumor cells did not achieve pCR in this study. These data may be critical to achieve pCR in neoadjuvant chemotherapy for operable breast cancer.

In pre-operative strategies for HER2-negative breast cancer, the proportion of ER-positive tumor cells is extremely important. If we distinguish ER-positive tumors into those with values of ER-positive cells less than $30 \%$ and those having values greater than or equal to $30 \%$, HER2-negative breast cancer consists of three groups: triple-negative breast cancers (TNBCs), ER low-positive tumors and ER highly positive tumors. Chemotherapy is the only treatment for TNBCs. It aims to reduce tumor size and prevent mastectomy, prevent tumor recurrence after surgery or obtain information regarding chemosensitivity in vivo. Chemotherapy is also a potent strategy for ER low-positive tumors. If the aim of neoadjuvant chemotherapy is to reduce tumor size and avoid mastectomy, the treatment must be useful for ER low-positive tumors despite the extremely low pCR rate. This is because neoadjuvant chemotherapy successfully reduces clinical tumor size (Table I). Hormonal therapy may be unsuccessful in treating ER low-positive breast cancer. However, postoperative additional hormonal therapy for ER low-positive breast cancer may prevent tumor recurrence as a more favorable prognosis for tumors with $1 \%$ positive-staining tumor cells when treated with tamoxifen has been reported in one study (23). In addition, neoadjuvant chemotherapy can be used for ER highly positive breast cancer as the treatment effectively reduced clinical tumor size avoiding mastectomy (Table II). Patients with values of ER-positive cells greater than or equal to $90 \%$ also achieved a good clinical response to neoadjuvant chemotherapy (data not shown). However, neoadjuvant hormonal therapy may be preferred if it reduces tumor size and mastectomy is avoided without cytotoxic chemotherapy. Moreover, surgery followed by adjuvant hormonal therapy may be a preferable alternative for treating operable breast cancer. This is because most patients with highly endocrineresponsive tumors do not require adjuvant chemotherapy.

The survival data revealed in this study were different from those of other studies. pCR following neoadjuvant chemotherapy was not statistically associated with improved DFS in our study; however, other studies have demonstrated a statistical difference (14). There was only a slight trend in improvement in the DFS of patients who achieved pCR in our study. Based on the DFS curve according to pCR, the difference may reach a significant value with an increase in the number of patients. In addition, we did not find a statistical difference in DFS or OS according to ER status, although other studies showed that patients with ER-negative tumors achieved a statistically worse DFS or OS (14). Conversely, our patients with ER-negative tumors showed a better prognosis probably as patients with HER2-positive tumors were not included among the ER-negative patients. In the event the data from other studies included HER2-positive patients treated with neoadjuvant chemotherapy without trastuzumab, HER2 disease may have lowered the survival curves.

It is difficult to explain why patients with ER-positive tumors rarely achieved pCR. To understand this phenomenon, we hypothesize that each ER-positive tumor cell is insensitive to cytotoxic chemotherapy. In other words, cytotoxic chemotherapy is effective only for ER-negative tumor cells. Our results showed that all postmenopausal patients achieving pCR were ER-negative (data not shown). In postmenopausal patients, the effectiveness of chemotherapy must be purely cytotoxic. Of all the pre-menopausal patients, the majority of patients with pCR were ER-negative, and only 2 patients with pCR were ER-positive (15 and 30\%). It is known that chemotherapy not only has a cytotoxic effect, but also a hormonal effect in premenopausal patients. This indicates that the ovarian function suppression induced by chemotherapy possibly encouraged the achievement of pCR in pre-menopausal ER-positive patients with few positive tumor cells.

We demonstrated a predictive significance of the proportion of ER-positive or PgR-positive tumor cells in neoadjuvant chemotherapy for operable HER2-negative breast cancer. ER- or PgR-negativity is a significant predictive factor to achieve pCR in multivariate analysis. Conversely, patients with more than $30 \%$ ER-positive tumor cells or more than $1 \%$ PgR-positive tumor cells may not achieve pCR. However, 
pre-menopausal patients with ER low-positive tumors may achieve pCR. In conclusion, most patients achieve favorable clinical responses to neoadjuvant chemotherapy for operable breast cancer regardless of the proportion of ER-positive or PgR-positive tumor cells.

\section{Acknowledgements}

The authors thank the staff members of the Department of Clinical Pathology at our hospital for the technical support.

\section{References}

1. Van der Hage JA, van de Velde CJ, Julien JP, Tubiana-Hulin M, Vandervelden $\mathrm{C}$ and Duchateau L: Preoperative chemotherapy in primary operable breast cancer: results from the European Organization for Research and Treatment of Cancer trial 10902. J Clin Oncol 19: 4224-4237, 2001.

2. Bear HD, Anderson S, Brown A, et al: The effect on tumor response of adding sequential preoperative docetaxel to preoperative doxorubicin and cyclophosphamide: preliminary results from the National Surgical Adjuvant Breast and Bowel Project Protocol B-27. J Clin Oncol 21: 4165-4174, 2003.

3. Fisher B, Bryant J, Wolmark N, et al: Effect of preoperative chemotherapy on the outcome of women with operable breast cancer. J Clin Oncol 16: 2672-2685, 1998.

4. Makris A, Powles TJ, Ashley SE, et al: A reduction in the requirements for mastectomy in a randomized trial of neoadjuvant chemoendocrine therapy in primary breast cancer. Ann Oncol 9: 1179-1184, 1998.

5. Mauriac L, MacGrogan G, Avril A, et al: Neoadjuvant chemotherapy for operable breast carcinoma larger than $3 \mathrm{~cm}$ : a unicentre randomized trial with a 124-month median followup. Institut Bergonie Bordeaux Groupe Sein (IBBGS). Ann Oncol 10: 47-52, 1999.

6. Rouzier R, Perou CM, Symmans WF, et al: Breast cancer molecular subtypes respond differently to preoperative chemotherapy. Clin Cancer Res 11: 5678-5685, 2005.

7. Rastogi P, Anderson SJ, Bear HD, et al: Preoperative chemotherapy: updates of National Surgical Adjuvant Breast and Bowel Project Protocols B-18 and B-27. J Clin Oncol 26: 778-785, 2008

8. Thomas E, Holmes FA, Smith TL, et al: The use of alternate, non-cross-resistant adjuvant chemotherapy on the basis of pathologic response to a neoadjuvant doxorubicin-based regimen in women with operable breast cancer: long-term results from a prospective randomized trial. J Clin Oncol 22: 2294-2302, 2004

9. Bonadonna G, Valagussa P, Brambilla C, et al: Primary chemotherapy in operable breast cancer: eight-year experience at the Milan Cancer Institute. J Clin Oncol 16: 93-100, 1998.

10. Kuerer HM, Newman LA, Smith TL, et al: Clinical course of breast cancer patients with complete pathologic primary tumor and axillary lymph node response to doxorubicin-based neoadjuvant chemotherapy. J Clin Oncol 17: 460-469, 1999.
11. Eltahir A, Heys SD, Hutcheon AW, et al: Treatment of large and locally advanced breast cancers using neoadjuvant chemotherapy. Am J Surg 175: 127-132, 1998.

12. Carey LA, Metzger R, Dees EC, et al: American Joint Committee on Cancer tumor-node-metastasis stage after neoadjuvant chemotherapy and breast cancer outcome. J Natl Cancer Inst 97: $1137-1142,2005$.

13. Hennessy BT, Hortobagyi GN, Rouzier R, et al: Outcome after pathologic complete eradication of cytologically proven breast cancer axillary node metastases following primary chemotherapy. J Clin Oncol 23: 9304-9311, 2005.

14. Ring AE, Smith IE, Ashley S, Fulford LG and Lakhani SR: Oestrogen receptor status, pathological complete response and prognosis in patients receiving neoadjuvant chemotherapy for early breast cancer. Br J Cancer 91: 2012-2017, 2004.

15. Burcombe RJ, Makris A, Richman PI, et al: Evaluation of ER, PgR, HER-2 and Ki-67 as predictors of response to neoadjuvant anthracycline chemotherapy for operable breast cancer. Br J Cancer 92: 147-155, 2005

16. Colleoni M, Bagnardi V, Rotmensz N, et al: Increasing steroid hormone receptor expression defines breast cancer subtypes non-responsive to preoperative chemotherapy. Breast Cancer Res Treat 116: 359-369, 2009.

17. Colleoni M, Viale G, Zahrieh D, et al: Expression of ER, PgR, HER1, HER2, and response: a study of preoperative chemotherapy. Ann Oncol 19: 465-472, 2008.

18. Colleoni M, Viale G, Zahrieh D, et al: Chemotherapy is more effective in patients with breast cancer not expressing steroid hormone receptors: a study of preoperative treatment. Clin Cancer Res 10: 6622-6628, 2004.

19. Hammond ME, Hayes DF, Dowsett M, et al: American Society of Clinical Oncology/College of American Pathologists guideline recommendations for immunohistochemical testing of estrogen and progesterone receptors in breast cancer. J Clin Oncol 28: 2784-2795, 2010

20. Buzdar AU, Ibrahim NK, Francis D, et al: Significantly higher pathologic complete remission rate after neoadjuvant therapy with trastuzumab, paclitaxel, and epirubicin chemotherapy: results of a randomized trial in human epidermal growth factor receptor 2-positive operable breast cancer. J Clin Oncol 23: 3676-3685, 2005

21. Eisenhauer EA, Therasse P, Bogaerts J, et al: New response evaluation criteria in solid tumours: revised RECIST guideline (version 1.1). Eur J Cancer 45: 228-247, 2009.

22. Kai K, Arima N, Miyayama H, Yamamoto Y, Iwase H and Nishimura R: Pathological lymph node involvement at surgery is a significant predictive factor of recurrence in locally advanced breast cancer treated with concomitant epirubicin-docetaxel neoadjuvant chemotherapy: a cohort study. Breast Cancer 16: 42-48, 2009.

23. Harvey JM, Clark GM, Osborne CK and Allred DC: Estrogen receptor status by immunohistochemistry is superior to the ligand-binding assay for predicting response to adjuvant endocrine therapy in breast cancer. J Clin Oncol 17: 1474-1481, 1999. 